\title{
The Emotional Perception Deficit in Social Phobia
}

\author{
Haoru $\mathrm{Li}^{1,}$, * \\ ${ }^{1}$ Pennsylvania State University, State College, PA, U.S \\ ${ }^{*}$ Corresponding author. Email: ${ }^{a} 1 \mathrm{hml5322@psu.edu}$
}

\begin{abstract}
This review article is mainly about the emotional perception in social phobia. When communication happens in a social group, the function of the emotional perception is to accurately capture other people's emotions. The emotional reactions can be trigger by the emotions perceived through vision, hearing, and touch. If you do not have an accurate emotional perception, you may not make a correct judgment on emotions, which is the biggest problem facing patients with social phobia. Researches on social phobia mainly focus on the role of self-focused attention in social phobia. Self-focused attention is defined as being aware of self-reference and being able to generate information internally. The review article discussed social phobia from the perspective of development. It was also found that the defects of emotion perception and the atypical neural mechanism defects of social phobia. Related literature has studied the patterns of reaction of patients with social phobia to specific emotions. Relevant works of literature have studied emotional perception and memory recall and studied the defects of emotional perception in development. Atypical was found in adults and children with social phobia. Relevant literature has studied the causes of neural mechanisms in patients with social phobia by eye-tracking test, electroencephalogram, and fMRI. Studies in the literature have found that the response of social phobia patients to feedback is also typical. Attention can change the anxiety of social phobia and reduce bias. The limitation of the studies is the studies only control the background factors. The studies only did the cross-sectional competitor. The sample size of the studies is too small. Future research needs to adjust limitations, carry out background classification, start the longitudinal investigation, and increase the sample size.
\end{abstract}

Keywords: Social Phobia, Emotional Perception, Anxiety, Self-image, Childhood.

\section{INTRODUCTION}

Emotional perception holds special importance. It's a process of interpretation and label [1]. The sensory system and some brain regions are used to decode emotional information for fast and efficient processing. Emotional perception is generally regarded as having three components: a subjective experience, physical change, and cognitive evaluation [1]. Through the information get by emotional perception can influence the emotion and give the appropriate response accurately. If there is a problem with emotional perception, the signal may be misinterpreted and cannot establish effective communication. How emotion can be perceived depends on the subjective consciousness of the individual. Social phobia, also known as social anxiety disorder, is an anxiety disorder characterized by anxiety in social situations and an impaired ability to function in daily life. The pathogenesis for social phobia is the fear of others evaluating themselves. By understanding the emotional perception of social phobia patients, more effective interventions can be developed to improve patients' fear of being evaluated. In this way, the interventions may help social phobia patients establish effective social relations with others.

At present, research on social phobia mainly focuses on the role of self-focused attention in social phobia. Selffocused attention is defined as being aware of selfreferential and can generate information internally [2]. Current studies suggest that self-focused attention is an important factor leading to disorder because the negative thoughts increased by self-focused attention will interfere with the performance of patients with social phobia. The studies suggested that self-focused attention itself does not have serious harm. Only in the case of excessive will it become pathological. Research shows that self-focused attention may lead to an increase in self-evaluation anxiety. This finding is aimed at all people, including people with social phobia and healthy subjects. However, the increase in self-anxiety will be more obvious in patients with a social phobia, leading to more anxiety. The study found that this situation can be improved. Using transference-focused psychotherapy (TFP) is to divert 
patients' attention with social phobia from the negative view to external events [3]. This psychotherapy will reduce the anxiety of patients and increase the positive view of themselves. If patients with social phobia can aim the self-focused attention on external events or positive views, patients' anxiety will be reduced. At present, most studies support the view that patients with social phobia feel anxious not based on other people's reactions but based on their impressions. Therefore, patients with a social phobia should focus on improving the anxiety produced when other people evaluate the patients.

The research on social phobia mainly solves the impact of self-focused attention on patients with social phobia. The negative thoughts caused by self-focused attention will aggravate the anxiety of patients with social phobia. Emotional perception is important in the sensory system. Emotional perception plays an important role in building effective social relationships, receiving and understanding signals, and other social functions. A social phobia is a group with defects in social function. But few studies have linked social phobia with emotional perception. This review will summarize the finding of related articles and fill the blanks in this area. If it can improve the emotional understanding of patients with social phobia, in this way, it may help patients with social phobia. It will discuss the relationship between emotional perception and the difficulty of social phobia from two aspects. The abnormality of emotional perception in a developmental perspective and atypical neural mechanism of the emotional perception.

\section{THE EMOTIONAL PERCEPTION DEFICIT IN A DEVELOPMENTAL PERSPECTIVE}

If the patients with social phobia have difficulty processing the emotional problem, they will likely influence their social life. It is important to understand whether the social phobia has a problem processing all the emotions or has a problem with the specific emotion. In the study of Mogg et al., to study the facial reaction time of patients with social phobia to emotion, they compared subjects in the social anxiety group and healthy people without known mental illness to be the control group [4]. The experimental condition included angry faces and happy faces, and neutral faces. The exposure time of the photos included $500 \mathrm{~ms}$ and $1250 \mathrm{~ms}$. They asked all the subjects to press the button to distinguish the different pictures. In the experimental condition of $500 \mathrm{~ms}$ exposure, the patients with social phobia had attention bias to angry faces; the healthy control group did not produce attention bias. In the $1250 \mathrm{~ms}$ experiment, patients with social phobia had attention bias to angry faces and have no problem processing the happy faces or the neutral faces [5]. Studies have shown that at $500 \mathrm{~ms}$, social phobia patients will be alert to social anxiety. The subjects with social phobia had a special reaction to angry faces during $500 \mathrm{~ms}$ and $1250 \mathrm{~ms}$. This means that people with social phobia cannot communicate effectively because they cannot receive signals accurately.

It is important to understand whether the problem with processing angry faces is started at which developmental stage. If the problem started when they were young, treatment could be used to intervene early. The experiment of Waters et al. compared children with social phobia (513 years of age) with healthy children on face recognition tests [6]. The experimental team used this study to verify the effect of attention bias in children with social phobia. The experiment required all the children participating in the test to quickly identify two groups of faces: angry and neutral face condition, happy and neutral face condition [7]. Attention bias is a well-established cognitive correlate of anxiety disorders. The results show that children with high social phobia are more likely to have attention bias to angry faces compared with healthy children. There is no difference between the data of children with social phobia and healthy children [7]. Comparing with healthy children, the children with social phobia have higher matching accuracy toward angry faces than happy faces. The bias toward the angry face does exist in childhood for the subjects with social phobia.

Facial emotional precessing is one kind of emotional perception. There are other aspects to perceiving the emotions, like recalling the memory, body language. Generally, memory recall can help the individual to summarize the experience. Individuals can make progress when they are facing a similar event. What if patients with social phobia also have negative feelings when recalling the memory under the stressful, it is essential to understand whether social phobia has difficulties in regulating their negative feelings. In the study of Schmitz et al., they reviewed the previous experiment that adults with social phobia mainly recalled a negative side in the distressful social event [8]. They investigated whether children with social phobia had the same problems as adults. The experiment asks the patients and health group to complete two tasks that can make them feel distressful: 1 speech task, 2 math tasks, and then all subjects have to complete a brief interview on mental images [9]. They used the Trier Social Stress Test to evaluate the performance of all the subjects. $2.5 \mathrm{~h}$ after the Trier Social Stress Test for Children (TSST-C), children with social phobia and adults with social phobia mainly recalled the negative part of that distressful social event [9]. Children with social phobia usually recall the negative memory about the judgment from others and negative selfevaluation. Children with social phobia had selfawareness, and they knew that their performance was worse than that of healthy controls, but the data did not change in subsequent experiments. The subjects with social phobia tend to recall the negative part of a stressful memory compared with the healthy control group. It might be due to the sensitive self-image which exists in childhood. 


\section{ATYPICAL NEURAL MECHANISM UNDERLYING THE EMOTIONAL PERCEPTION DEFICIT}

Now It is acknowledged that there is some problem for the individual to processing the emotion. However, it is still necessary to find out which specific pattern is unusual for emotional perception. In Boll et al.'s experiment, they asked the patients with social phobia and healthy subjects as controls to do the eye-tracking experiment [10]. Each participant was asked to look at 40 face photos (all the features of stimuli were controlled). Participants were asked to classify the emotion in the photos as quickly as possible by pressing keys on the computer keyboard. The experimenters recorded the eye fixation for all the subjects in the picture and the duration of their eyes' fixation [11]. The study found that the main observation area of the patients with social phobia was the eyes, and the observation time was higher than that of the healthy group [12]. The study also found a delayed attentional in eye gaze direction, which suggests a differential processing time in patients and controls. Social phobia patients have problems processing emotions because they tend to have atypical in processing the facial comparing with healthy people.

It is important to investigate whether subjects with social phobia neural mechanisms underlying the emotional perception are the same as typically developing controls. The brain region also plays an important role in precept emotion. For example, the amygdala is the brain tissue that processes emotions, recognizes and regulates emotions The study of Danti et al. mainly focused on the facial perception of patients with social phobia using fMRI [13]. The task is designed to be a recall task, demonstrate a photo first, then show a target photo. Asked the patient if the two photos were the same face. The subjects were asked to task during the fMRI sessions [13]. The subjects had to use the same response button to indicate whether successive pictures were the same. The brain activities of social patients are atypical comparing with the healthy control group. These areas included the bilateral fusiform gyrus, the right superior temporal sulcus (R-STS), and the left amygdala [14]. The left amygdala has a stronger activity for the subjects with social phobia. The connectivity of these brain areas is also different from the healthy control group. The activity of the left amygdala for patients with social phobia is different comparing with healthy people. The connectivity is also different when processing facial information.

FMRI can observe the overall abnormalities in brain areas, and event-related potentials can observe real-time dynamics. There was a negative correlation between the intensity of the electroencephalogram (EEG)waveform, and P6 represents the violation of the expected value. The bigger the gap between what people find and what they expect, the stronger P6 will be. The study of Moser et al. mainly studies the unique and comprehensive effects of social phobia and depression on ambiguous social situations using the electroencephalogram (EEG) [15]. By monitoring the current on the brain's surface, event-related potentials can determine the specific time when the brain signals [16]. In the experiment, the amplitude at the center of the head of all the subjects was monitored when they read sentences. There were neutral, negative, and positive situations in the sentences. Patients with social phobia have weaker amplitude at the head center when neutral words appear because they are not surprised by neutral words. The results show differences in the neutral voltage of event-related brain potentials in $600 \mathrm{~ms}$ (P6). The difference between P6 and neutral endpoint reaction time was still significant. The decreased P6 in the socially phobic groups found here reflects their distraction by negative self-imagery. The study found a lack of positive bias across patients diagnosed with social phobia, a positive interpretation bias in the healthy control group [17]. The accuracy and reaction time did not reveal any differences between the two groups in the neutral voltage. The reaction times of processing the positive feedback and negative feedback are different compare to the healthy subjects. The study also found that the presence of a negative bias characterized the depressed group. Therefore, social phobia patients do not focus on positive feedback. They pay too much attention to the negative side of their self-image. People with social phobia do not make changes, according to the feedback.

If emotional perception plays an important role in social phobia, then relevant training will alleviate some symptoms. In the study of Heeren et al., they studied whether two kinds of attention training can reduce the anxiety of social phobia and whether attention training in any direction can reduce anxiety by means of a selfassessment report [18]. Participants watched two stimuli that were presented in two areas of the computer screen. After the picture disappeared, a probe immediately replaced one of the stimuli. This means that the subjects' attention is shifted when they respond to the probe faster than when they replace the non-threat stimuli. In the experiment, the subjects were told to make a speech according to negative emotions and recorded the skin conductance as the basis of physical response. Studies have shown that anxiety in patients with social phobia decreases during attention training [19]. It supports that attention training can affect anxiety symptoms. Attention training reduced self-reported distress. It shows that patients' anxiety symptoms with social phobia can be reduced after completing emotional perception-related training

\section{LIMITATION AND FUTURE DIRECTION}

The current research is a cross-sectional study, aiming at the same age group of patients with social phobia and healthy people for comparison. Social phobia children and healthy children, social phobia adults and healthy adults. A cross-sectional study can determine the difference 
between the same kind but cannot find out the emotional development process. The current experiments are all conducted in the laboratory, mainly reading words and observing facial expressions. This kind of experiment is far away from real life. In daily life, the right situation will happen, and the variables will not be controlled as in the laboratory. There may be stress in life and different results in the laboratory. The study only controlled the background information of social phobia patients rather than studying their impact. This kind of experiment cannot effectively analyze the differences of social phobia patients. The sample size of the research is too small. Some experiments only have 30 subjects. It is difficult to accurately analyze the situation of patients with social phobia. Although there is attention training, there is no experiment associated with the training.

In future research, they need to focus more on the longitudinal study. Tracking this kind of study can help to understand the development status of emotional perception. For example, in the development process of self-awareness, what factors lead to the change of selfawareness. Future experiments should add real scenes, such as inviting friends or family members to participate in the experiment. The subjects were no longer faced with machines but with people with independent thinking. In this case, the experimental results and the performance in daily life are more appropriate. In future research, it can be classified according to socioeconomic. Future studies can investigate the performance of social phobia patients with rich families and the performance social phobia patients with higher education have. In future experiments, they need to increase the sample size. If there are enough experimental data, they can accurately understand the situation of patients with social phobia.

\section{CONCLUSION}

The function of emotional perception is to process other people's emotions accurately through the sense of vision, hearing, touch, etc. In contrast, patients with social phobia cannot accurately capture others' emotions because they fear being judged by others when having communications with others. Current studies have found that people with social phobia have a special responsibility to angry faces. Social phobia patients' bias to angry faces appeared in childhood. Social phobia patients will be very sensitive to the self-image in childhood, which will lead to easy recall of negative memory. People with social phobia have difficulty dealing with emotions in part because they do not process facial information the same aspects healthy people do. There are differences in brain activity between patients with social phobia and healthy people. People with social phobia do not expect positive feedback. There is no specific classification according to the background, no vertical comparison, and less experimental data. Future research needs to distinguish social phobia patients in different environments according to the background, understand the development process of social phobia through longitudinal comparison, and increase the experimental data.

\section{REFERENCES}

[1] Spurr, J. M., \& Stopa, L. (2002). Self-focused attention in social phobia and social anxiety. Clinical psychology review, 22(7), 947-975.

[2] Kimbrel, N. A. (2008). A model of the development and maintenance of generalized social phobia. Clinical psychology review, 28(4), 592-612.

[3] Diemer, J., Alpers, G. W., Peperkorn, H. M., Shiban, Y., \& Mühlberger, A. (2015). The impact of perception and presence on emotional reactions: a review of research in virtual reality. Frontiers in psychology, 6, 26.

[4] Mogg, K., Philippot, P., \& Bradley, B. P. (2004). Selective attention to angry faces in clinical social phobia. Journal of abnormal psychology, 113(1), 160.

[5] Diagnostic, A. P. A. (1994). statistical manual of mental disorders. Washington. DC: American Psychiatric Association, 4.

[6] Waters, A. M., Mogg, K., Bradley, B. P., \& Pine, D. S. (2011). Attention bias for angry faces in children with social phobia. Journal of Experimental Psychopathology, 2(4), 475-489.

[7] Bradley, B. P., Mogg, K., Falla, S. J., \& Hamilton, L. R. (1998). Attentional bias for threatening facial expressions in anxiety: Manipulation of stimulus duration. Cognition \& emotion, 12(6), 737-753.

[8] Schmitz, J., Krämer, M., Blechert, J., \& TuschenCaffier, B. (2010). Post-event processing in children with social phobia. Journal of abnormal child psychology, 38(7), 911-919.

[9] Abbott, M. J., \& Rapee, R. M. (2004). Post-event rumination and negative self-appraisal in social phobia before and after treatment. Journal of abnormal psychology, 113(1), 136.

[10] Boll, S., Bartholomaeus, M., Peter, U., Lupke, U., \& Gamer, M. (2016). Attentional mechanisms of social perception are biased in social phobia. Journal of anxiety disorders, 40, 83-93.

[11] Akiyama, T., Kato, M., Muramatsu, T., Umeda, S., Saito, F., \& Kashima, H. (2007). Unilateral amygdala lesions hamper attentional orienting triggered by gaze direction. Cerebral Cortex, 17(11), 2593-2600.

[12] Amir, N., Foa, E. B., \& Coles, M. E. (1998). Automatic activation and strategic avoidance of 
threat-relevant information in social phobia. Journal of abnormal psychology, 107(2), 285.

[13] Danti, S., Ricciardi, E., Gentili, C., Gobbini, M. I., Pietrini, P., \& Guazzelli, M. (2010). Is social phobia a "mis-communication" disorder? Brain functional connectivity during face perception differs between patients with social phobia and healthy control subjects. Frontiers in Systems Neuroscience, 4, 152.

[14] American Psychiatric Association, A. (1980). Diagnostic and statistical manual of mental disorders (Vol. 3). Washington, DC: American Psychiatric Association.

[15] Moser, J. S., Huppert, J. D., Foa, E. B., \& Simons, R. F. (2012). Interpretation of ambiguous social scenarios in social phobia and depression: evidence from event-related brain potentials. Biological Psychology, 89(2), 387-397.

[16] Abramson, L. Y., Metalsky, G. I., \& Alloy, L. B. (1989). Hopelessness depression: A theory-based subtype of depression. Psychological review, 96(2), 358.

[17] Anderson, C. A., \& Arnoult, L. H. (1985). Attributional style and everyday problems in living: Depression, loneliness, and shyness. Social cognition, 3(1), 16-35.

[18] Heeren, A., Reese, H. E., McNally, R. J., \& Philippot, P. (2012). Attention training toward and away from threat in social phobia: Effects on subjective, behavioral, and physiological measures of anxiety. Behaviour research and therapy, 50(1), 3039.

[19] American Psychiatric Association. (1994). DSM-IV: Diagnostic and statistical manual of mental disorders. 